\title{
Method of Lines for the Chiral Nonlinear Schrödinger Equation
}

\author{
K. S. AL-Basyouni, M. S. Ismail \\ Department of Math., College of Science, King Abdulaziz University, Jeddah, Saudi Arabia \\ Email: msismail@kau.edu.sa, kalbasyouni@kau.edu.sa
}

How to cite this paper: AL-Basyouni, K.S. and Ismail, M.S. (2020) Method of Lines for the Chiral Nonlinear Schrödinger Equation. Applied Mathematics, 11, 447-459. https://doi.org/10.4236/am.2020.116032

Received: April 10, 2020

Accepted: May 31, 2020

Published: June 3, 2020

Copyright $\odot 2020$ by author(s) and Scientific Research Publishing Inc. This work is licensed under the Creative Commons Attribution International License (CC BY 4.0).

http://creativecommons.org/licenses/by/4.0/

\section{(c) (i) Open Access}

\begin{abstract}
In this paper, we solve chiral nonlinear Schrodinger equation (CNSE) numerically. Two numerical methods are derived using the explicit Runge-Kutta method of order four and the linear multistep method (Predictor-Corrector method of fourth order). The resulting schemes of fourth order accuracy in spatial and temporal directions. The CNSE is non-integrable and has two kinds of soliton solutions: bright and dark soliton. The exact solutions and the conserved quantities of CNSE are used to display the efficiency and robustness of the numerical methods we derived. Interaction of two bright solitons for different parameters is also displayed.
\end{abstract}

\section{Keywords}

Method of Lines, Linear Multistep Method, Chiral Soliton, Interaction of Two Solitons

\section{Introduction}

The chiral nonlinear Schrödinger equation (CNSE) [1] [2] we are going to study is given by

$$
i \frac{\partial \psi}{\partial t}+\frac{1}{2} \frac{\partial^{2} \psi}{\partial x^{2}}+i \lambda\left(\psi^{*} \frac{\partial \psi}{\partial x}-\psi \frac{\partial \psi^{*}}{\partial x}\right) \psi=0,-\infty<x<\infty
$$

where $t$ is time, $x$ is spatial coordinate, $\psi(x, t)$ is a complex-valued function, and $\lambda$ is a nonlinear coupling constant appears through derivative coupling. This kind of nonlinearity is also known as the current density, unlike the case of cubic nonlinearity which is also known as the Kerr nonlinearity. The Chiral nonlinear Schrödinger is a non-integrable equation by the classical method of Inverse Scattering method. The single bright soliton solution of Equation (1) is given by [1] [2] 


$$
\psi(x, t)=A \operatorname{sech}(\beta(x-v t)) \exp (i(v x+\omega t)),
$$

where

$$
\beta=A \sqrt{2 \lambda v} \text { and } \omega=\frac{v\left(2 \lambda A^{2}-v\right)}{2},
$$

$A$ is the amplitude of the soliton, $\beta$ is the inverse width of the soliton, $V$ is the soliton velocity, and $\omega$ is the wave number. The dark soliton solution for (1) exists for $\lambda v<0$, and has the form

$$
\psi(x, t)=A \tanh (\beta(x-v t)) \exp (i(v x+\omega t))
$$

where

$$
\beta=A \sqrt{-2 \lambda v} \text { and } \omega=\frac{v\left(4 \lambda A^{2}-v\right)}{2} .
$$

Thus Equation (1) has bright or dark solitons that are given by (2) and (4), respectively, depending on the sign of $\lambda v$. This phenomenon makes the solitons chiral. Equation (1) has at least four conserved quantities [1] [2], namely

$$
\begin{gathered}
I_{1}(t)=\int_{-\infty}^{\infty}|\psi(x, t)|^{2} \mathrm{~d} x, \\
I_{2}(t)=i \int_{-\infty}^{\infty}\left(\bar{\psi}(x, t) \psi_{x}(x, t)-\psi(x, t) \bar{\psi}_{x}(x, t)\right) \mathrm{d} x, \\
I_{3}(t)=\int_{-\infty}^{\infty}\left[\lambda|\psi(x, t)|^{4}+2 i\left(\bar{\psi}(x, t) \psi_{x}(x, t)-\psi(x, t) \bar{\psi}_{x}(x, t)\right)\right] \mathrm{d} x,
\end{gathered}
$$

and

$$
I_{4}=\int_{-\infty}^{\infty}\left[\lambda\left|\psi_{x}(x, t)\right|^{2}+2 i\left(\bar{\psi}(x, t) \psi_{x}(x, t)-\psi(x, t) \bar{\psi}_{x}(x, t)\right)+\lambda|\psi(x, t)|^{6}\right] \mathrm{d} x
$$

Due to the exponential decay of the bright soliton solution (2) when $|x| \rightarrow \infty$, the conserved quantities (6)-(9) are well defined. By using (2), the exact values of the conserved quantities are

$$
\begin{aligned}
& I_{1}=\frac{\beta}{\lambda v}, I_{2}=\frac{2 \beta}{\lambda v}, I_{3}=\frac{4 A^{2}}{3 \beta}\left(\lambda A^{2}+6 v\right), \\
& I_{4}=\frac{4 A^{2}}{3 b}\left(15 v^{2}+5 \beta^{2}+40 \lambda v A^{2}+8 \lambda^{2} A^{4}\right) .
\end{aligned}
$$

The conserved quantities (6)-(9) using dark soliton solution (4) are not well defined due to the nonzero boundary condition as $|x| \rightarrow \infty$.

By assuming $\psi(x, t)=u(x, t)+i v(x, t)$, where $u(x, t), v(x, t)$ are real functions, the CNSE (1) can be written as the nonlinear coupled system [3] [4] [5] as

$$
\begin{aligned}
& \frac{\partial u}{\partial t}+\frac{1}{2} \frac{\partial^{2} v}{\partial x^{2}}+2 \lambda\left(u \frac{\partial v}{\partial x}-v \frac{\partial u}{\partial x}\right) v=0, \\
& \frac{\partial v}{\partial t}-\frac{1}{2} \frac{\partial^{2} u}{\partial x^{2}}-2 \lambda\left(u \frac{\partial v}{\partial x}-v \frac{\partial u}{\partial x}\right) u=0 .
\end{aligned}
$$

The resulting system obtained (11)-(12) can be displayed in a matrix vector form as 


$$
\frac{\partial \boldsymbol{w}}{\partial t}+\frac{1}{2} A \frac{\partial^{2} \boldsymbol{w}}{\partial x^{2}}+G(\boldsymbol{w}) A \boldsymbol{w}=\mathbf{0}
$$

where

$$
\boldsymbol{w}=\left[\begin{array}{l}
u \\
v
\end{array}\right], \quad A=\left(\begin{array}{cc}
0 & 1 \\
-1 & 0
\end{array}\right), \quad G(\boldsymbol{w})=2 \lambda\left(u \frac{\partial v}{\partial x}-v \frac{\partial u}{\partial x}\right)
$$

There are many theoretical and numerical studies in the literature about the Nonlinear Schrödinger Equations (NLS). Most of these works are motivated to single NLS and the coupled NLS (see [3] [4] [5] and reference therein). However, up to the authors knowledge, only a few numerical studies for the chiral nonlinear Schrodinger NLS. In [6], a conservative finite difference schemes for the chiral nonlinear schrodinger equation using finite difference method, three different schemes of second order accuracy are derived, a nonlinear implicit finite difference where we have to solve a nonlinear block tridigonal systems at each time step which is expensive to some extent, a linearization approach is used to overcome this difficulty and to obtain, two linearly implicit finite difference schemes. In this work, we are going to present numerical schemes of fourth order accuracy in both direction using method of lines. The method of lines has been used by several authors. A Method of lines is used to find the numerical solution of the Kortweg-de Vries Equation [7]. The method of lines solution of the regularized long-wave equation using Runge-Kutta time discretization method presented in [8]. The method of lines for solution of the one-dimensional wave equation subject to an integral conservation condition presented in [9]. Application of the method of lines for solving the KdV-Burger equation, two methods are used to solve this equation MOL and the Adomian decomposition method the results reveal that, both methods are comparable [10]. A generalization of the method of lines for the numerical solution of the coupled forced vibration of beams presented in [11]. In this work, we are going to solve the chiral nonlinear Schrodinger equation using method of lines, which can be described in two major steps.

Step 1: We convert the partial differential equation into a system of first order ordinary differential equation by approximating the space derivatives in the CNSE using fourth order finite difference approximation order.

Step 2: We solve the ordinary differential system obtained in Step 1 using Runge-Kutta method of fourth order and Predictor Corrector method of fourth order.

Several Numerical examples single and interaction of two solitons will be investigated to confirm the efficiency of the derived schemes.

The paper is organized as follows: In Section 2, two methods of lines are derived to solve the chiral NLS (1). In Section 3, some numerical results single soliton and interaction of two solitons to check the efficiency of the proposed schemes. Finally, the conclusion is given in Section 4 .

\section{Numerical Methods}

We will consider the numerical solution of the nonlinear system (10)-(11) in a 
finite interval $\left[x_{L}, x_{R}\right]$. We assume $x_{m}=x_{L}+m h$, where $m=1,2, \cdots, M-1$, and $h$ is called the space grid size, also we assume $t_{n}=n k, k$ is the time step size. We denote the exact and numerical solutions at the grid point $\left(x_{m}, t_{n}\right)$ by $\left(\boldsymbol{w}_{m}^{n}, v_{m}^{n}\right)$ and $\left(\boldsymbol{U}_{m}^{n}, V_{m}^{n}\right)$, respectively. Also we approximate the space derivatives using the fourth order approximation [12].

$$
\begin{gathered}
\frac{\partial u\left(x_{m}\right)}{\partial x}=\frac{1}{12 h}\left[U_{m-2}-8 U_{m-1}+8 U_{m+1}-U_{m+2}\right] \\
\frac{\partial^{2} u\left(x_{m}\right)}{\partial x^{2}}=\frac{1}{12 h^{2}}\left[-U_{m-2}+16 U_{m-1}-30 U_{m}+16 U_{m+1}-U_{m+2}\right] .
\end{gathered}
$$

By using these approximations into (11)-(12), we will get the first order ordinary differential coupled system

$$
\begin{aligned}
& \dot{U}_{m}+\frac{1}{24 h^{2}}\left[-U_{m-2}+16 U_{m-1}-30 U_{m}+16 U_{m+1}-U_{m+1}\right] \\
& +\frac{\lambda}{6 h}\left(U_{m}\left[V_{m-2}-8 V_{m-1}+8 V_{m+1}-V_{m+2}\right]\right. \\
& \left.-V_{m}\left[U_{m-2}-8 U_{m-1}+8 U_{m+1}-U_{m+2}\right]\right) V_{m}=0, \\
& U_{-1}=U_{0}=0, U_{M}=U_{M+1}=0, \text { for } m=1,2, \cdots, M-1 \\
& \dot{V}_{m}-\frac{1}{24 h^{2}}\left[-U_{m-2}+16 U_{m-1}-30 U_{m}+16 U_{m+1}-U_{m+1}\right] \\
& -\frac{\lambda}{6 h}\left(U_{m}\left[V_{m-2}-8 V_{m-1}+8 V_{m+1}-V_{m+2}\right]\right. \\
& \left.-V_{m}\left[U_{m-2}-8 U_{m-1}+8 U_{m+1}-U_{m+2}\right]\right) U_{m}=0, \\
& V_{-1}=V_{0}=0, V_{M}=V_{M+1}=0, \text { for } m=1,2, \cdots, M-1,
\end{aligned}
$$

The numerical solution of the coupled system (17)-(18) can be obtained by using the Runge-Kutta method of fourth order or by using a linear multistep method in the following manner. First we write the previous system (17)-(18) in the coupled form

$$
\begin{aligned}
& \dot{U}_{m}=F\left(U_{m}, V_{m}\right), m=1,2, \cdots, M-1 \\
& \dot{V}_{m}=F\left(U_{m}, V_{m}\right), m=1,2, \cdots, M-1
\end{aligned}
$$

The basic idea of the method of lines is to replace the spatial derivatives in the partial differential equation with algebraic approximations. Once this is carried out, the spatial derivatives are no longer stated explicitly in terms of the spatial independent variables. Thus, in effect we have a coupled system of ordinary differential equations that approximate the original PDE. Two methods for solving the ODEs coupled system (19)-(20) are presented; Runge-Kutta method of fourth order and the fourth order Predictor-Corrector methods. The details of these methods will be given next.

\subsection{Method of Lines Using Fourth Order Runge-Kutta Method}

In this method we use one-step method, to achieve high accuracy it is typically necessary to use multistage method, where the intermediate values of the solu- 
tion and its derivatives are generated and used within a single time step. To apply this approach, we use Runge-Kutta method of fourth order to solve the ordinary differential coupled systems (ODEs) [12].

First we define

$$
\boldsymbol{U}^{n}=\left[U_{1}^{n}, U_{2}^{n}, \cdots, U_{M-1}^{n}\right]^{t} \text { and } \boldsymbol{V}^{n}=\left[V_{1}^{n}, V_{2}^{n}, \cdots, V_{M-1}^{n}\right]^{t},
$$

and then we define the Runge-Kutta method as follows

$$
\begin{aligned}
& \boldsymbol{U}^{n+1}=\boldsymbol{U}^{n}+\frac{1}{6}\left[K_{11}+2 K_{12}+2 K_{13}+K_{14}\right] \\
& \boldsymbol{V}_{m}^{n+1}=\boldsymbol{V}^{n}+\frac{1}{6}\left[K_{21}+2 K_{22}+2 K_{23}+K_{24}\right]
\end{aligned}
$$

where

$$
\begin{gathered}
K_{11}=h \boldsymbol{F}\left(\boldsymbol{U}^{n}, V_{m}^{n}\right), K_{21}=h \boldsymbol{G}\left(\boldsymbol{U}^{n}, \boldsymbol{V}^{n}\right), \\
K_{12}=h \boldsymbol{F}\left(\boldsymbol{U}^{n}+\frac{1}{2} K_{11}, \boldsymbol{V}^{n}+\frac{1}{2} K_{21}\right), \\
K_{22}=h \boldsymbol{G}\left(\boldsymbol{U}^{n}+\frac{1}{2} K_{11}, \boldsymbol{V}^{n}+\frac{1}{2} K_{21}\right), \\
K_{13}=h \boldsymbol{F}\left(\boldsymbol{U}^{n}+\frac{1}{2} K_{12}, \boldsymbol{V}^{n}+\frac{1}{2} K_{22}\right), \\
K_{23}=h \boldsymbol{G}\left(\boldsymbol{U}^{n}+\frac{1}{2} K_{12}, \boldsymbol{V}^{n}+\frac{1}{2} K_{22}\right), \\
K_{14}=h \boldsymbol{F}\left(\boldsymbol{U}^{n}+K_{13}, \boldsymbol{V}^{n}+K_{23}\right), \\
K_{24}=h \boldsymbol{G}\left(\boldsymbol{U}^{n}+K_{13}, \boldsymbol{V}^{n}+K_{23}\right)
\end{gathered}
$$

Note to apply the Runge-Kutta method, we need to evaluate eight vector functions at each time step.

\subsection{Method of Lines Using Linear Multistep Method of Fourth Order}

One approach to use the linear multistep method is to use a predictor-corrector method, in which an explicit Adams-Basforth method of fourth order is used to predict a value for numerical solution $U^{(p)}$, and then the Adams-Moulton of method of fourth order is used to correct this value. This is done by using $U^{(p)}$ on the right hand side of the Adams -Moulton method inside the function evaluation, so that the Adams-Moulton is no longer implicit. Now to apply this method in our problem, we execute the following steps.

\section{Step 1 Starting Values}

Use the explicit Runge-Kutta method of fourth order

$$
\begin{aligned}
& U_{m}^{n+1}=U_{m}^{n}+\frac{1}{6}\left[K_{11}+2 K_{12}+2 K_{13}+K_{14}\right] \\
& V_{m}^{n+1}=V_{m}^{n}+\frac{1}{6}\left[K_{21}+2 K_{22}+2 K_{23}+K_{24}\right]
\end{aligned}
$$


where

$$
\begin{gathered}
K_{11}=h \boldsymbol{F}\left(\boldsymbol{U}^{n}, V_{m}^{n}\right), K_{21}=h \boldsymbol{G}\left(\boldsymbol{U}^{n}, \boldsymbol{V}^{n}\right), \\
K_{12}=h \boldsymbol{F}\left(\boldsymbol{U}^{n}+\frac{1}{2} K_{11}, \boldsymbol{V}^{n}+\frac{1}{2} K_{21}\right), \\
K_{22}=h \boldsymbol{G}\left(\boldsymbol{U}^{n}+\frac{1}{2} K_{11}, \boldsymbol{V}^{n}+\frac{1}{2} K_{21}\right), \\
K_{13}=h \boldsymbol{F}\left(\boldsymbol{U}^{n}+\frac{1}{2} K_{12}, \boldsymbol{V}^{n}+\frac{1}{2} K_{22}\right), \\
K_{23}=h \boldsymbol{G}\left(\boldsymbol{U}^{n}+\frac{1}{2} K_{12}, \boldsymbol{V}^{n}+\frac{1}{2} K_{22}\right), \\
K_{14}=h \boldsymbol{F}\left(\boldsymbol{U}^{n}+K_{13}, \boldsymbol{V}^{n}+K_{23}\right), \\
K_{24}=h \boldsymbol{G}\left(\boldsymbol{U}^{n}+K_{13}, \boldsymbol{V}^{n}+K_{23}\right)
\end{gathered}
$$

to calculate the missing values

$$
\boldsymbol{U}^{n}, \boldsymbol{V}^{m}, \text { for } n=1,2,3 \text { and } m=1,2, \cdots, M-1
$$

Step 2: Predictor (Adams Bashforth of fourth order)

$$
\begin{aligned}
\boldsymbol{U}^{(p)}= & \boldsymbol{U}^{n}+\frac{k}{24}\left[55 \boldsymbol{F}\left(\boldsymbol{U}^{n}, \boldsymbol{V}^{n}\right)-59 \boldsymbol{F}\left(\boldsymbol{U}^{n-1}, \boldsymbol{V}^{n-1}\right)\right. \\
& \left.+37 \boldsymbol{F}\left(\boldsymbol{U}^{n-2}, \boldsymbol{V}^{n-2}\right)-9 \boldsymbol{F}\left(\boldsymbol{U}^{n-3}, \boldsymbol{V}^{n-3}\right)\right] \\
\boldsymbol{V}^{(p)}= & \boldsymbol{V}^{n}+\frac{k}{24}\left[55 \boldsymbol{G}\left(\boldsymbol{U}^{n}, \boldsymbol{V}^{n}\right)-59 \boldsymbol{G}\left(\boldsymbol{U}^{n-1}, \boldsymbol{V}^{n-1}\right)\right. \\
& \left.+37 \boldsymbol{G}\left(\boldsymbol{U}^{n-2}, \boldsymbol{V}^{n-2}\right)-9 \boldsymbol{G}\left(\boldsymbol{U}^{n-3}, \boldsymbol{V}^{n-3}\right)\right]
\end{aligned}
$$

for $n=3,4, \cdots, N$.

Step 3: Corrector (Adams Moulton of fourth order)

$$
\begin{aligned}
\boldsymbol{U}^{n+1}= & \boldsymbol{U}^{n}+\frac{k}{24}\left[9 \boldsymbol{F}\left(\boldsymbol{U}^{p}, \boldsymbol{V}^{p}\right)+19 \boldsymbol{F}\left(\boldsymbol{U}^{n}, \boldsymbol{V}^{n}\right)\right. \\
& \left.-5 \boldsymbol{F}\left(\boldsymbol{U}^{n-1}, \boldsymbol{V}^{n-1}\right)+\boldsymbol{F}\left(\boldsymbol{U}^{n-2}, \boldsymbol{V}^{n-2}\right)\right] \\
\boldsymbol{V}^{n+1}= & \boldsymbol{V}^{n}+\frac{k}{24}\left[9 \boldsymbol{G}\left(\boldsymbol{U}^{p}, \boldsymbol{V}^{p}\right)+19 \boldsymbol{G}\left(\boldsymbol{U}^{n}, \boldsymbol{V}^{n}\right)\right. \\
& \left.-5 \boldsymbol{G}\left(\boldsymbol{U}^{n-1}, \boldsymbol{V}^{n-1}\right)+\boldsymbol{G}\left(\boldsymbol{U}^{n-2}, \boldsymbol{V}^{n-2}\right)\right]
\end{aligned}
$$

for $n=3,4, \cdots, N$.

Note that step 1 is used to find the starting values using Runge-Kutta method of fourth order. We conclude that, at each time step, the numerical solution obtained by executing Step 2 and Step 3. Note that we need only four function evaluation at each time step, which is almost half of the Runge-Kutta method (21)-(22).

\section{Numerical Results}

In this section, we will test the efficiency of the numerical schemes we derived, 
by considering different numerical tests. Trapezoidal rule is used to calculate the conserved quantities. Define the error norms

$$
\begin{gathered}
L_{\infty}(u)=\max _{1 \leq m \leq M-1}\left|u_{m}^{n}-U_{m}^{n}\right| \\
L_{2}(u)=\sqrt{\left[h \sum_{m=1}^{M-1}\left|u_{m}^{n}-U_{m}^{n}\right|^{2}\right] .}
\end{gathered}
$$

\subsection{Bright Soliton Solution}

To study the behavior of the single bright soliton solution, we choose the initial condition

$$
\psi(x, 0)=A \operatorname{sech}(\beta x) \exp (i v x)
$$

together with the homogenous Dirichlet boundary conditions $\psi(x, 0)=0$ at $x=x_{L}, x_{R}$. The following set of parameters are used

$$
\begin{aligned}
& x_{L}=-50.0, x_{R}=50.0 .0, h=0.1, k=0.001, A=0.5, \\
& \lambda=0.5, v=0.5, t=0,1,2, \cdots, 20 .
\end{aligned}
$$

Table 1 and Table 2 display the errors and the conserved quantities for our proposed schemes. The numerical results are highly accurate and conserved the conserved quantities exactly. The two methods produced almost the same results (both fourth order methods in space and time directions). In Figure 1, we display the motion of the single bright solitons for $t=0,0.5, \cdots, 10$. We have noticed that the cpu execution time required for producing the results in Table 1, Table 2 are 4.14 and 2.65 seconds respectively...

Table 1. Single bright soliton using Runge-Kutta method $(\mathrm{cpu}=4.14)$.

\begin{tabular}{ccccccc}
\hline $\mathrm{T}$ & $I_{1}$ & $I_{2}$ & $I_{3}$ & $I_{4}$ & $L_{\infty}$ & $L_{2}$ \\
\hline 0 & 1.414214 & 1.413330 & 2.944511 & 3.044373 & - & - \\
5 & 1.414214 & 1.413330 & 2.944511 & 3.044373 & 0.00000 & 0.000001 \\
10 & 1.414214 & 1.413330 & 2.944511 & 3.044373 & 0.000001 & 0.000001 \\
15 & 1.414214 & 1.413330 & 2.944511 & 3.044373 & 0.000001 & 0.000002 \\
20 & 1.414214 & 1.413330 & 2.944511 & 3.044373 & 0.000001 & 0.000003 \\
\hline
\end{tabular}

Table 2. Single bright soliton using predictor-corrector method $(\mathrm{cpu}=2.656)$.

\begin{tabular}{ccccccc}
\hline $\mathrm{T}$ & $I_{1}$ & $I_{2}$ & $I_{3}$ & $I_{4}$ & $L_{\infty}$ & $L_{2}$ \\
\hline 0 & 1.414214 & 1.413330 & 2.944511 & 3.044373 & - & - \\
5 & 1.414214 & 1.413330 & 2.944511 & 3.044373 & 0.00000 & 0.000001 \\
10 & 1.414214 & 1.413330 & 2.944511 & 3.044373 & 0.000001 & 0.000001 \\
15 & 1.414214 & 1.413330 & 2.944511 & 3.044373 & 0.000001 & 0.000002 \\
20 & 1.414214 & 1.413330 & 2.944511 & 3.044373 & 0.000001 & 0.000003 \\
\hline
\end{tabular}




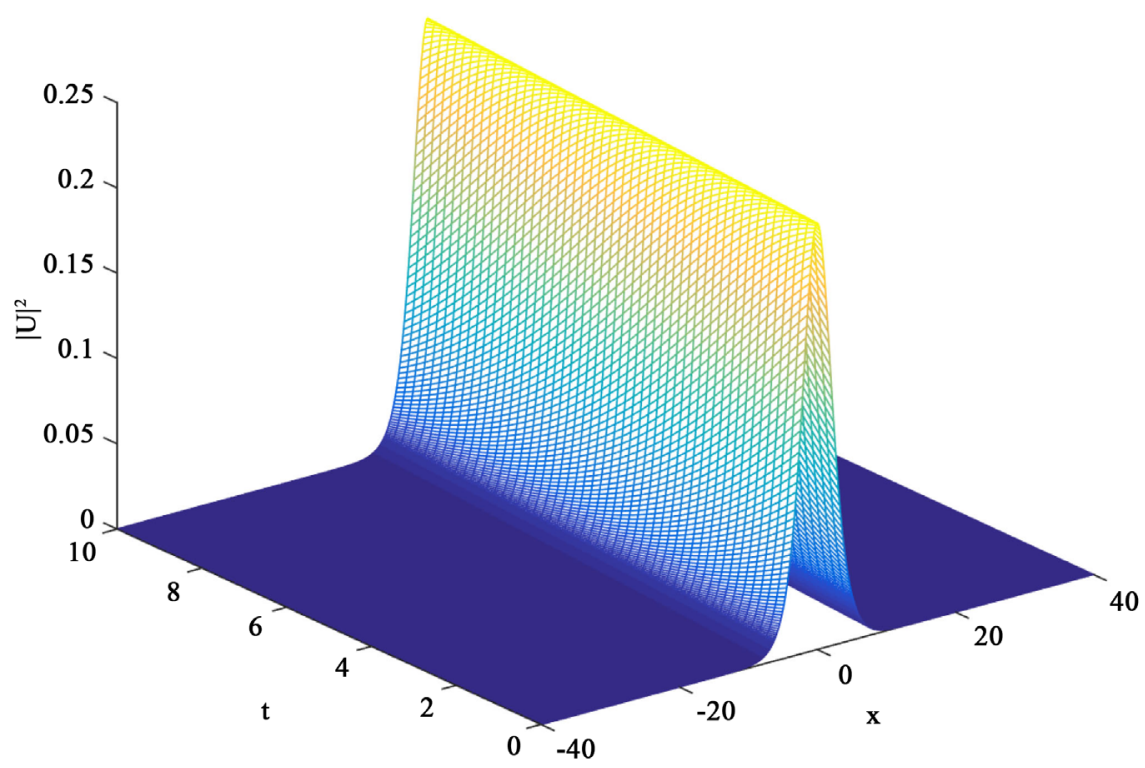

Figure 1. Single Bright soliton: $A=0.5, \lambda=0.5, v=0.5$.

\subsection{Interaction of Two Bright Solitons}

In non-integrable equations, stable solitons may exist, but their collisions are generally inelastic. To study the interaction behavior of the two bright solitons in the chiral nonlinear Schrodinger, we choose the initial condition [13]

$$
\psi(x, 0)=\psi_{1}(x, 0)+\mathrm{e}^{\delta} \psi_{2}(x, 0),
$$

where

$$
\begin{gathered}
\psi_{1}(x, 0)=A_{1} \operatorname{sech}\left(\beta_{1}\left(x-x_{1}\right)\right) \exp \left(i\left(v_{1}\left(x-x_{1}\right)\right)\right), \\
\psi_{2}(x, 0)=A_{2} \operatorname{sech}\left(\beta_{1}\left(x-x_{2}\right)\right) \exp \left(i\left(v_{2}\left(x-x_{2}\right)\right)\right),
\end{gathered}
$$

and $\delta \in[-\pi, \pi]$ is the phase difference between the solitons. The initial condition represents the sum of two well separated single bright solitons. To study the interaction scenario the following parameters are selected

$$
\begin{aligned}
& h=0.1, k=0.001, x_{l}=-50, x_{1}=-x_{2}=20, A_{1}=1.0, \\
& v_{1}=1.0, A_{2}=0.5, v_{2}=0.1, \lambda=0.5, t=0,1,2, \cdots, 80 .
\end{aligned}
$$

The conserved quantities of the interaction scenario are given in Table 3. We have noticed that the numerical results are highly accurate and the exact conservation of $I_{1}$. In Figure 2 and Figure 3, we display the interaction scenario, and we have noticed how the two solitons approach each other and the faster soliton emerges to the slower soliton. Eventually, the two original solitons leave the interaction region with their original shape.

In Figures 4-9, we display the interaction scenario of two solitons for different parameters. Different scenario obtained, the interaction are similar to those produced by conventional nonlinear Schrodinger equation dynamics. Also we have noticed in Figure 6 and Figure 7, fussion of solitons is observed by choosing $\delta=0.4 \pi$. 


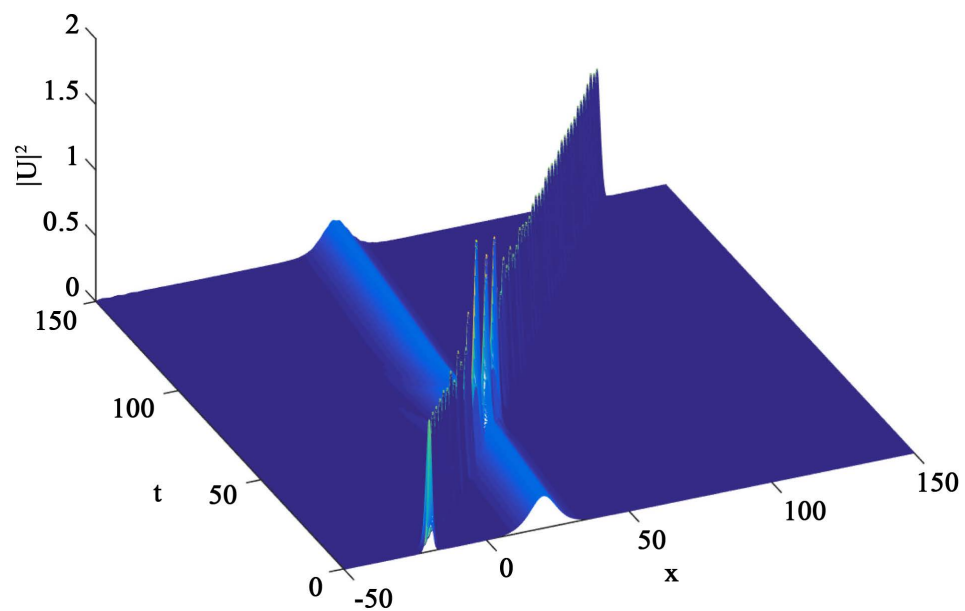

Figure 2. Interaction of two solitons: $A_{1}=1, A_{2}=0.5, v_{1}=1, v_{2}=0.1, \lambda=0.5, \delta=0$.

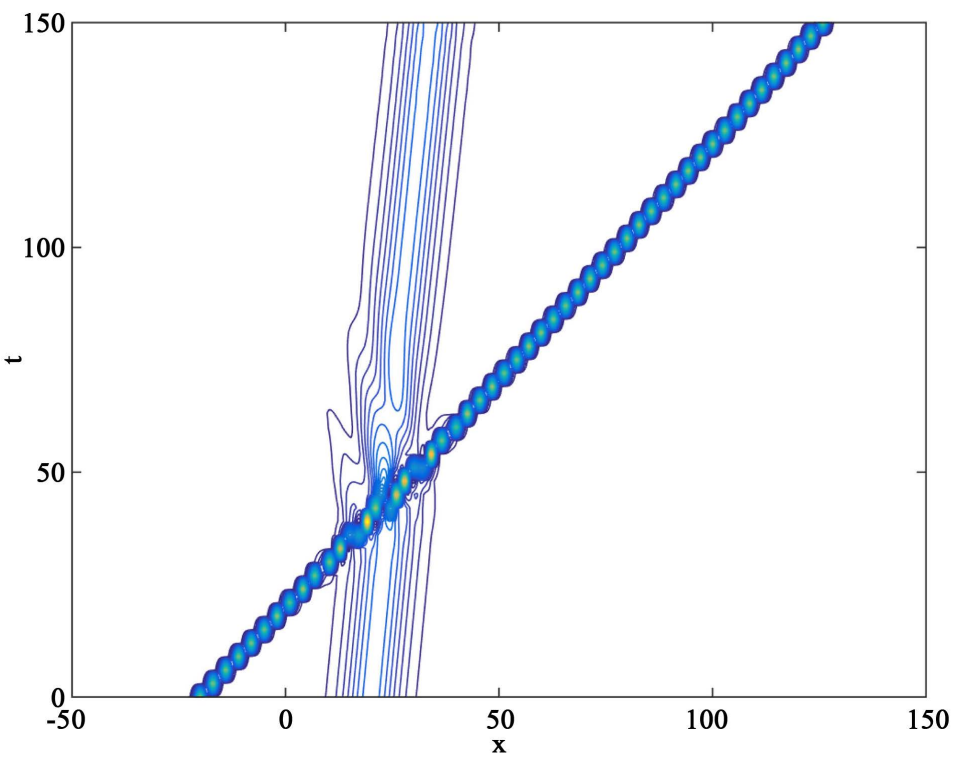

Figure 3. Interaction of two solitons: $A_{1}=1, A_{2}=0.5, v_{1}=1, v_{2}=0.1, \lambda=0.5, \delta=0$.

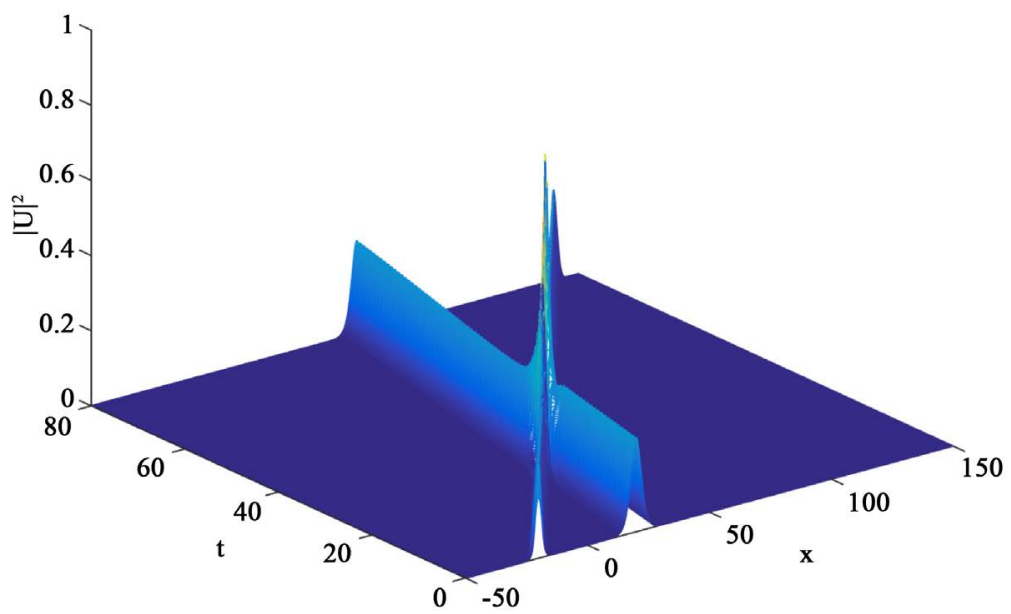

Figure 4. Interaction of two solitons: $A_{1}=0.5, A_{2}=0.5, v_{1}=2, v_{2}=0.5, \lambda=0.5, \delta=\pi$. 


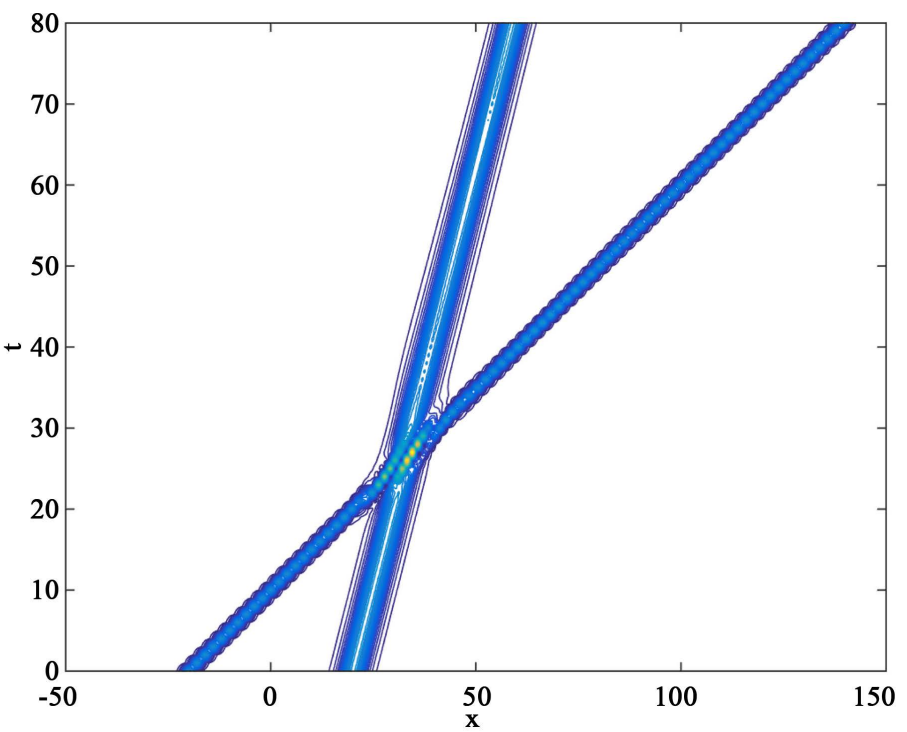

Figure 5. Interaction of two solitons: $A_{1}=0.5, A_{2}=0.5, v_{1}=2, v_{2}=0.5, \lambda=0.5, \delta=\pi$.

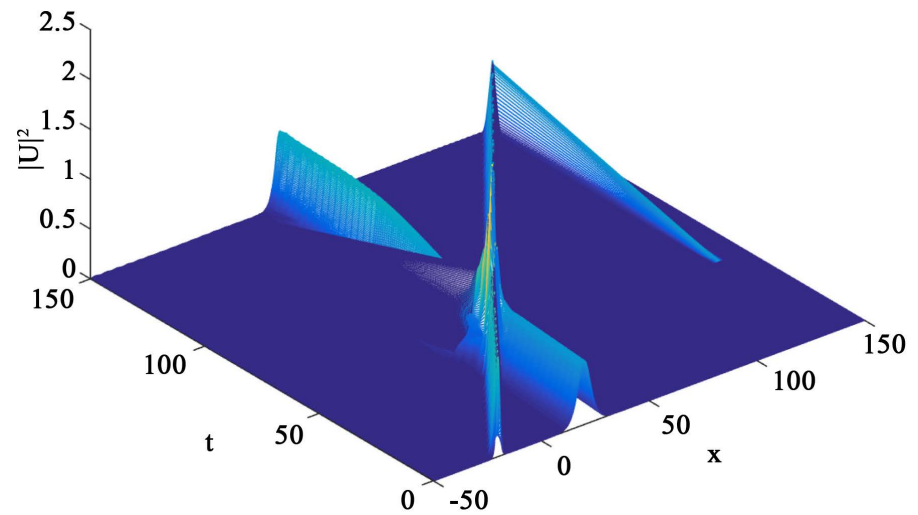

Figure 6. Interaction of two solitons: $A_{1}=1.0, A_{2}=0.8, v_{1}=1, v_{2}=0.1, \lambda=0.5, \delta=0.4 \pi$.

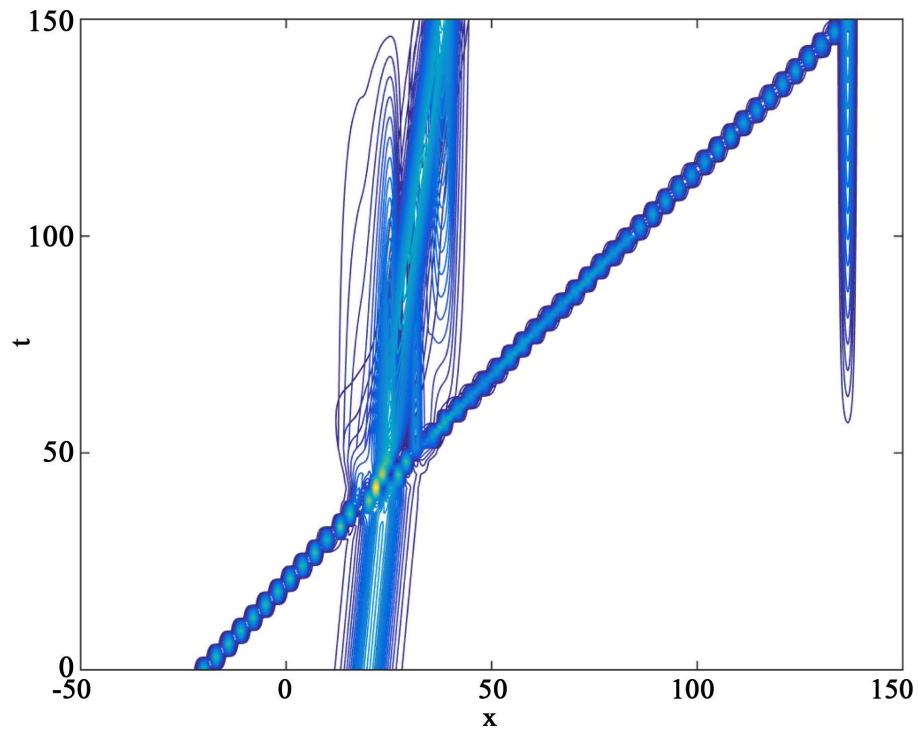

Figure 7. Interaction of two solitons: $A_{1}=1.0, A_{2}=0.8, v_{1}=1, v_{2}=0.1, \lambda=0.5, \delta=0.4 \pi$. 


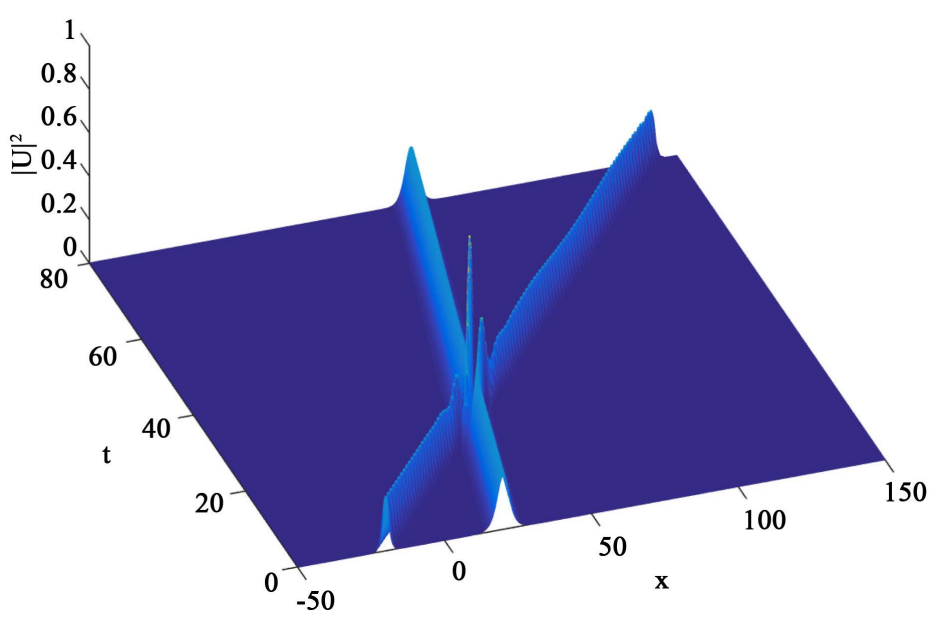

Figure 8. Interaction of two solitons: $A_{1}=0.5, A_{2}=0.5, v_{1}=2, v_{2}=0.5, \lambda=0.5, \delta=\pi$.

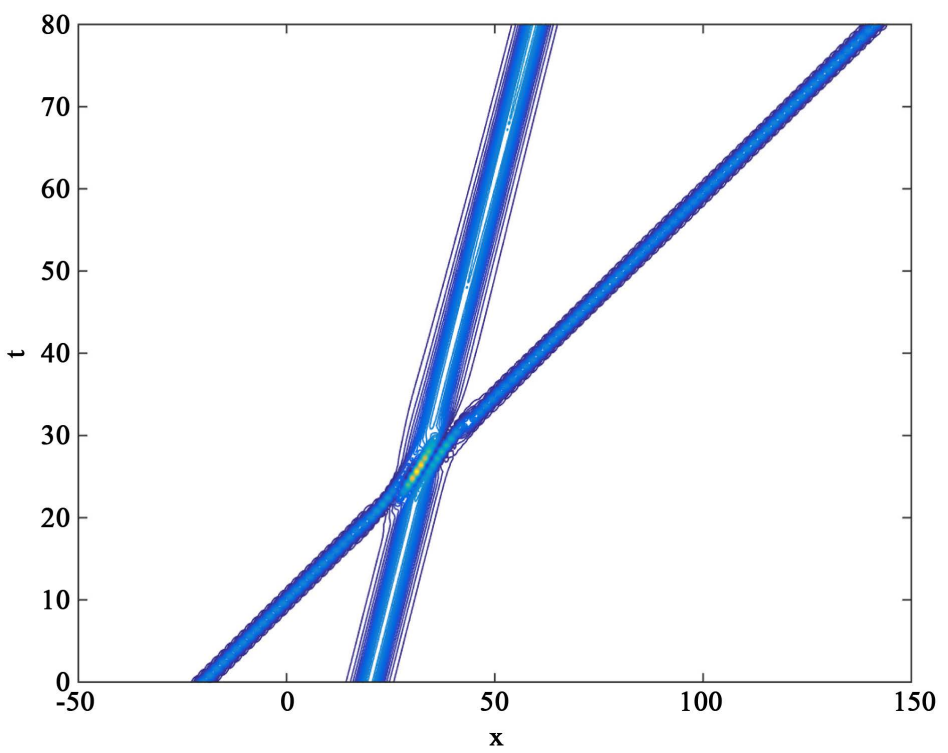

Figure 9. Interaction of two solitons: $A_{1}=0.5, A_{2}=0.5, v_{1}=2, v_{2}=0.5, \lambda=0.5, \delta=\pi$.

Table 3. Interaction of two bright solitons using Runge-Kutta method.

\begin{tabular}{ccccc}
\hline $\mathrm{t}$ & $I_{1}$ & $I_{2}$ & $I_{3}$ & $I_{4}$ \\
\hline 0 & 5.164771 & 4.618162 & 10.169401 & 10.881909 \\
20 & 5.164771 & 4.575337 & 10.105110 & 10.811868 \\
40 & 5.164771 & 4.551480 & 10.070448 & 10.725963 \\
60 & 5.164771 & 4.503832 & 9.998313 & 10.703303 \\
80 & 5.164769 & 4.479468 & 9.499313 & 10.661822 \\
\hline
\end{tabular}

\subsection{Single Dark Soliton}

To study the behavior of the single dark soliton solution, we choose the initial condition

$$
\psi(x, 0)=A \tanh (\beta x) \exp (i v x)
$$


and use the Dirichlet boundary conditions at $x=x_{L}, x_{R}$ which are extracted from the exact solution (Boundary conditions are nonzero). The following set of parameters are used

$$
\begin{aligned}
& x_{L}=-50.0, x_{R}=50, h=0.1, k=0.001, A=0.5, \\
& \lambda=-0.5, v=0.5, t=0,0.4,0.8, \cdots, 20 .
\end{aligned}
$$

In Table 4 , we calculate the $L_{\infty}$ and $L_{2}$ error norms and the conserved quantity $I_{1}$, we found that the proposed methods are highly accurate. The simulation of the single dark soliton displayed in Figure 10 for $t=0,0.4, \cdots, 20$. The cpu execution time is 4.14 section, which is the same as the bright soliton.

\section{Conclusion}

The method of lines is used to solve the Chiral nonlinear Schrodinger equation, using two methods of fourth order accuracy in both directions space and time. The numerical results using single soliton, and the interaction of two solitons of the CNSE confirmed the efficiency, reliability, and accuracy of the present methods. Concerning the cpu time required for execution, the merit goes for the Predictor-Corrector method. We can conclude that, the methods presented in this paper can be applied to similar type of equations, like nonlinear Schrodinger and coupled nonlinear Schrodinger equations.

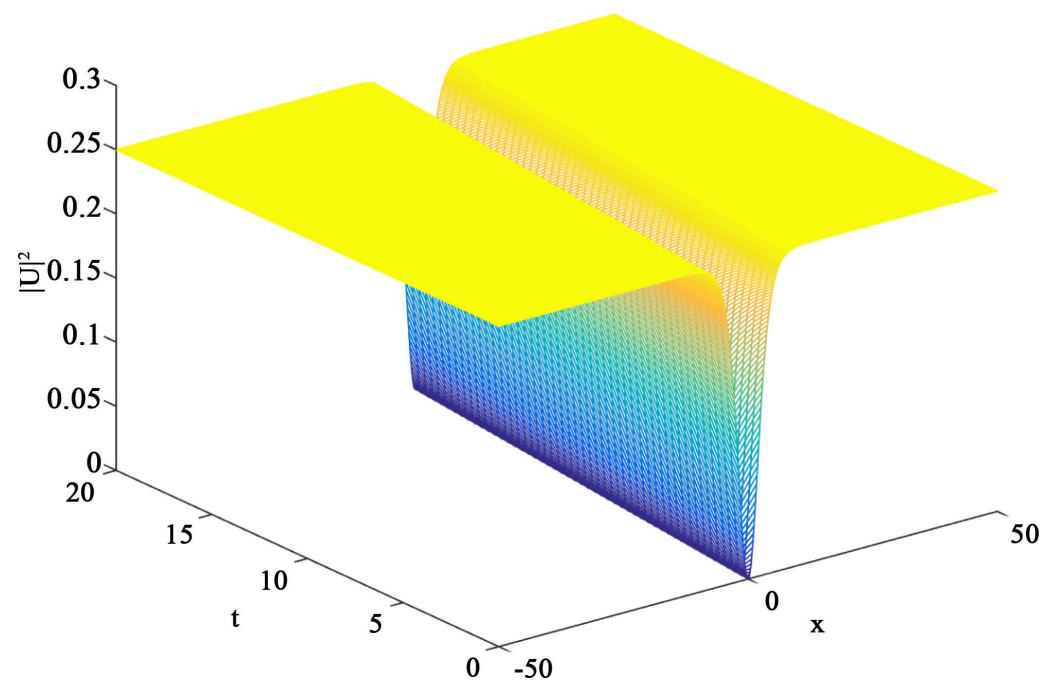

Figure 10. Dark soliton: with $A=0.5, \lambda=-0.5, v=0.5$.

Table 4. Single Dark soliton using Runge-Kutta method (cpu = 4.14).

\begin{tabular}{cccc}
\hline $\mathrm{T}$ & $I_{1}$ & $L_{\infty}$ & $L_{2}$ \\
\hline 0 & 23586786 & 0.000000 & 0.000000 \\
5 & 23.585682 & 0.000062 & 0.00000 \\
10 & 23.585674 & 0.000065 & 0.000001 \\
15 & 23.585675 & 0.000067 & 0.000001 \\
20 & 23585677 & 0.000066 & 0.000001
\end{tabular}




\section{Conflicts of Interest}

The authors declare no conflicts of interest regarding the publication of this paper.

\section{References}

[1] Biswas, A. (2009) Chiral Solitons in $1+2$ Dimensions. International Journal of Theoretical Physics, 48, 3403-3409. https://doi.org/10.1007/s10773-009-0145-4

[2] Biswas, A. (2009) Perturbation of Chiral Solitons. Nuclear Physics B, 806, 457-461. https://doi.org/10.1016/j.nuclphysb.2008.05.023

[3] Ismail, M.S. and Taha, T.R. (2001) Numerical Simulation of Coupled Nonlinear Schrödinger Equation. Mathematics and Computers in Simulation, 56, 547-562. https://doi.org/10.1016/S0378-4754(01)00324-X

[4] Ismail, M.S. and Alamri, S.Z. (2004) Highly Accurate Finite Difference Method for Coupled Nonlinear Schrödinger Equation. International Journal of Computer Mathematics, 81, 333-351. https://doi.org/10.1080/00207160410001661339

[5] Ismail, M.S. and Taha, T.R. (2007) A Linearly Implicit Conservative Scheme for the Coupled Nonlinear Schrödinger Equation. Mathematics and Computers in Simulation, 74, 302-311. https://doi.org/10.1016/j.matcom.2006.10.020

[6] Ismail, M.S., Al-Basyouni, K.S. and Aydin, A. (2015) Conservative Finite Difference Schemes for the Chiral Nonlinear Schrodinger Equation. Boundary Value Problems, 2015, Article No. 89. https://doi.org/10.1186/s13661-015-0350-4

[7] Schiesser, W.E. (1994) Method of Lines Solution of the Kortweg-de Vries Equation. Computers \& Mathematics with Applications, 28, 147-154.

https://doi.org/10.1016/0898-1221(94)00190-1

[8] Bakodah, H.O. and Banaja, M.A. (2013) The Method of Lines Solution of the Regularized Long-Wave Equation Using Runge-Kutta Time Discretization Method. Mathematical Problems in Engineering, 2013, Article ID: 804317 https://doi.org/10.1155/2013/804317

[9] Shakeri, F. and Dehghan, M. (2008) The Method of Lines for Solution of the One-Dimensional Wave Equation Subject to an Integral Conservation Condition. Computers and Mathematics with Applications, 56, 2175-2188. https://doi.org/10.1016/j.camwa.2008.03.055

[10] El Sadat, R. and Ali, M.R. (2017) Application of the Method of Lines for Solving the KdV-Burger Equation. BISKA NTMSCT, 12, 39-51.

[11] Sarker, P. and Chakravarty, U.K. (2020) A Generalization of the Method of Lines for the Numerical Solution of Coupled, Forced Vibration of Beams. Mathematics and Computers in Simulation, 170, 115-142.

https://doi.org/10.1016/j.matcom.2019.10.011

[12] LeVeque, R.J. (2007) Finite Difference Methods for Ordinary and Partial Differential Equations, Steady-State and Time Dependent Problems. Siam, Philadelphia. https://doi.org/10.1137/1.9780898717839

[13] Dingwell, R.D., Edmonds, M.J., Helm, J.L., Malomed, B.A. and Ohberg, P. (2018) Non-Integrable Dynamics of Matter-Wave Solitons in a Density-Dependent Gauag Theory. New Journal of Physics, 20, Article ID: 043004.

https://doi.org/10.1088/1367-2630/aab29e 